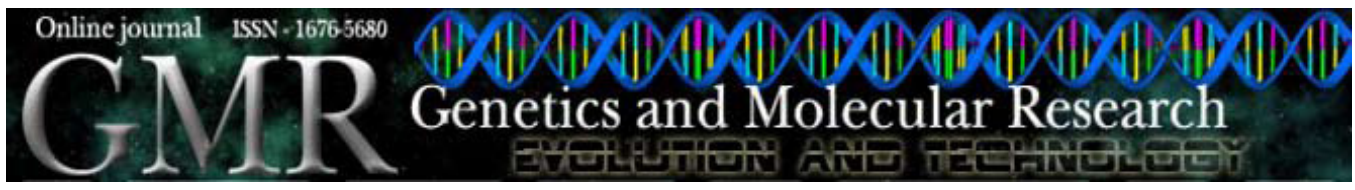

\title{
Mapping of major quantitative trait loci for economic traits of silkworm cocoon
}

\author{
Z. Lie ${ }^{1,2}$, L. Cheng ${ }^{1}$, D. Fang-yin ${ }^{1}$ and F. Shou-min ${ }^{2}$ \\ ${ }^{1}$ Department of Sericulture and Biotechnology, \\ Key Sericultural Laboratory of Agricultural Ministry, \\ Southwest University, Chongqing, China \\ ${ }^{2}$ College of Life Sciences, China West Normal University, \\ Nanchong, Sichuan, China \\ Corresponding author: L. Cheng \\ E-mail: lucheng@swu.edu.com
}

Genet. Mol. Res. 9 (1): 78-88 (2010)

Received August 25, 2009

Accepted October 21, 2009

Published January 19, 2010

\begin{abstract}
The quantitative trait loci (QTLs) associated with cocoon traits in silkworms were mapped in 44 individuals of a backcross of Dazao females with hybrid $\mathrm{F}_{1}$ males; the hybrid males were from females of inbred $\mathrm{C}_{100}$ strain, which have white cocoons and superior cocoon traits, crossed with males of inbred strain Dazao, which have green cocoons and inferior cocoon traits. Nineteen putative major QTLs of silkworm cocoon traits, five QTLs of whole cocoon weight, four QTLs of cocoon shell weight, six QTLs of pupa weight, and four QTLs of cocoon shell rate were scattered across nine linkage groups. The variances explained by QTLs for whole cocoon weight, cocoon shell weight, pupa weight, and cocoon shell rate were 51.0, 73.69, 51.80 , and $59.52 \%$, respectively. The numbers of major QTLs
\end{abstract}


with contributions above $10 \%$ for these traits were two, three, two, and four, respectively.

Key words: Bombyx mori; Economic traits; Silkworm cocoon; Composite interval mapping; Quantitative trait loci

\section{INTRODUCTION}

The objective of animal and plant breeding is to improve economic characters and to increase production. Classical methods of animal and plant breeding with phenotypic markers have made a significant contribution to their production and quality improvement since Sax (1923), but they have been slow in targeting complex traits such as yield, quality or resistance to unfavorable environments. One of the causes is that the number of phenotypic markers in animal and plant species has been insufficient for the requirements of animal and plant breeding and that phenotypic markers change easily with environment. The advent of molecular marker technology has brought flourish and powerful tools for animal and plant breeders to get more markers of animal and plant species. Polygenic characters, which were very difficult to analyze using traditional animal and plant breeding methods, can now be tagged firsthand using molecular markers (DNA markers). Therefore, molecular markers could locate and follow the numerous interacting genes that determine a complex trait. Quantitative trait locus (QTL) linkage mapping could provide a more direct method for selecting desirable genes via their linkage to easily detectable molecular markers (Tanksley et al., 1989). Hence, many QTL maps for various animals and plant species have been constructed, for example, QTL for production traits in chickens (Hansen et al., 2005), QTL mapping in pigs (Rothschild et al., 2007), and QTL mapping of potato (Bradshaw, 2008), and they are highly helpful for improving products of animal and plant and for exploring animal and plant breeding methods.

The silkworm (Bombyx mori L., $\mathrm{N}=28$ ), domesticated over the last 5000 years from a wild progenitor Bombyx mandarina (Zhou, 1958), is an important livelihood for subsistence farmers engaged in silk production in many countries. For more silk production and fine silk, many silkworm breeding methods have been developed, but these methods have not completely fulfilled the objective of silkworm breeding. MAS (markerassisted selection) technology (Thoday and Boam, 1961) has brought prosperity and has provided powerful tools, but the phenotypic markers have been insufficient for the MAS necessary. In the early 1980s, the advent of molecular marker technology (Saiki et al., 1992) opened up a new way for silkworm breeding. MAS methods were not only the number more than that of phenotypic markers in the traditional breeding, but could select traits in molecular heritance. Many molecular genetic maps of silkworm have been constructed since 1990 (Goldsmith, 1991; Li et al., 2000; Tan et al., 2001; Miao et al., 2005; Zhang et al., 2008). The MAS methods could promote breeding result, but a molecular genetic map or a QTL map was necessary to construction. With the development of polymorphic markers for many species, current research interest is to partition genetic variation to individual QTL in the genome as well as interaction among them (Zeng et al., 1999). The improvement of silkworm properties, including silk quality and quantity, is a constant target for silk production. Therefore, the construction of a QTL map of silkworm 
cocoon traits would not only improve silkworm breeding methods, but could also accelerate the achievement of objectives in silkworm breeding.

\section{MATERIAL AND METHODS}

\section{Mapping population}

The $\mathrm{BC}_{1}$ of mapping population from Dazao female crossed with hybrid $\mathrm{F}_{1}$ male, which inbred strain $\mathrm{C}_{100}$ with white cocoon female and its cocoon traits better crossed with inbred strain Dazao with green cocoon male and its cocoon traits worse (the crossing experiments were performed at the Institute of Silkworm and Mulberry, Southwest University, China), were employed to construct a genetic map. The $44 \mathrm{BC}_{1}$ pupas consisting of 11 green cocoon females and males and 11 white cocoon females and males, and anchoring the cocoon color gene in the backcross population, were randomly selected as materials; their important economic traits for the whole cocoon weight, cocoon shell weight, cocoon shell rate, and weight of pupae, were investigated and employed to a construct molecular linkage map.

\section{DNA extraction and detection of AFLP markers}

Template DNA was extracted with one third of each pupa, and the method used in this study consulted the methods of Williams et al. (1990) and Xia et al. (1998). Genome DNA of two parents, gene pool of 10 sib hybrid $\mathrm{F}_{1}$, and 44 individuals of backcrossed generation were used for detection of amplified fragment length polymorphism (AFLP) markers. The AFLP marker detection was according to Zabeau and Vos (1992), Vos et al. (1995), and Wan and Tan (1999). Two amplification reactions were carried out in a Thermolyne I thermocycler.

A total of 100 ng genomic DNA was digested with PstI and TaqI restriction enzymes, and then double-stranded adapters of Pst I and TaqI were ligated to the ends of the fragments employing $T_{4}$ DNA ligase in $4 \mu \mathrm{L}$ digested genomic DNA. Ligated DNA fragments were used as templates for preamplification. The primers of preamplification were complementary to the adapters with one additional selective nucleotide. The preamplification products diluted 8-fold were used as DNA templates of selective amplification with three additional selective nucleotides. The second amplification products were separated by $6 \%$ denaturing polyacrylamide gel electrophoresis. Electrophoresis was performed with an electrophoresis instrument in $1 \mathrm{X}$ TBE running buffer at constant power $(80 \mathrm{~W})$ for $3 \mathrm{~h} 20 \mathrm{~min}$ at $50^{\circ} \mathrm{C}$. Finally, the bands were revealed by silver staining (Promega silver staining kit).

\section{RESULTS}

\section{Construction of molecular linkage map}

Forty-four individuals of a backcrossed population $\left(\mathrm{BC}_{1}\right)$ that was derived from a cross between $\mathrm{C}_{100}$ and Dazao were selectively amplified by 96 of 144 primer pairs (combining 12 Pst I and $12 \mathrm{Taq}$ I primers). In total, 5204 polymorphic bands were amplified by the 96 primer pairs, and the rate of polymorphic bands was $61 \%$. Based on the $\chi^{2}$ test, $34 \%$ of polymorphic bands were in accordance with the segregation ratio of 1:1 (significance 
level, 0.05). The number of amplification bands was from 31 to 155, and the average of amplification bands for one set of primers was 89 .

A total of 1744 polymorphic bands conforming the ratio of 1:1 were utilized to construct a linkage map employing the QTXb19 (version 0.29) software. A total of 814 loci among 1744 bands were scattered across 35 linkage groups; the length of the linkage map was 12,896 $\mathrm{cM}$, and the distance between two markers was $2.3 \sim 47.7 \mathrm{cM}$, with the average distance between two markers being $16.7 \mathrm{cM}$. In addition, silkworm has 28 classical linkage groups, whereas in this study the number of linkage groups exceeded 28. It showed that some linkage groups probably linked with each other, but there was no specific marker to combine the linkage groups in our data, indicating that it needs to be substantiated further. Otherwise, the other markers could not locate their linkage group, so the efficiency of the mapped loci is only $46.7 \%$.

\section{QTL analysis of cocoon traits}

Quantitative traits of cocoon were composed of whole cocoon weight, cocoon shell weight, cocoon shell rate, and pupa weight, so those were analyzed at the level of the whole genome, employing the composite interval mapping software WinQTLCart2.0 (Wang et al., 2002). On the basis of the backcross information criterium standard, QTLs were scanned every $2 \mathrm{cM}$, and 19 QTLs were detected, where their ratio of contribution to additive effect was estimated (Table 1). Each QTL of scanning for four traits is illustrated in Figures 1-4, and the QTL's locations on AFLP molecular genetic map were displayed (Figure 5). The QTLs of the four important quantitative traits were detected as 5, 4, 6, and 4, respectively, whereas major QTLs with the ratios of contribution exceeding $10 \%$ were 2, 3, 2, and 4, respectively.

\begin{tabular}{|c|c|c|c|c|c|c|c|}
\hline Trait & Locus & $\begin{array}{l}\text { Linkage } \\
\text { group }\end{array}$ & Marker interval & $\begin{array}{l}\text { Confidence } \\
\text { interval }(\mathrm{cM})\end{array}$ & $\begin{array}{l}\text { Likelihood } \\
\text { ratio statistic }\end{array}$ & Additive & $\begin{array}{c}\text { Variance } \\
\text { explained }(\%)\end{array}$ \\
\hline \multirow[t]{5}{*}{ Cocoon weight } & qCW-4 & 4 & LP10T11-78, LP10T11-77 & $1143.3-1158$ & 19.31 & 1.571 & 6.2 \\
\hline & $\mathrm{qCW}-10$ & 10 & LP3T6-64, LP4T5-8 & $429.2-445.2$ & 21.36 & 1.803 & 10.9 \\
\hline & qCW-11 & 11 & LP6T1-102, LP11T12-9, LP11T12-15 & $44.6-76.8$ & 12.98 & -1.538 & 6.0 \\
\hline & qCW-15 & 15 & LP7T7-38, LP7T7-37 & $121.9-132.8$ & 13.95 & -1.299 & 4.7 \\
\hline & qCW-33 & 33 & LP3T6-5, LP5T8-22 & $226.5-236.5$ & 36.90 & 2.560 & 23.2 \\
\hline \multirow[t]{4}{*}{ Cocoon shell weight } & qSW-4 & 4 & LP11T4-19, LP5T12-45 & $1320.2-1340.4$ & 13.37 & -3.581 & 8.17 \\
\hline & qSW-10 & 10 & LP3T9-18, LP3T6-64 & $401.5-415.5$ & 27.23 & 5.67 & 17.76 \\
\hline & qSW-12 & 12 & LP7T12-13, LP7T11-9 & $88.5-103.8$ & 18.29 & 4.19 & 10.68 \\
\hline & qSW-33 & 33 & LP3T6-5, LP5T8-22 & $226.5-236.5$ & 43.21 & 7.27 & 37.08 \\
\hline \multirow[t]{7}{*}{ Pupa weight } & qPW-4 & 4 & LP12T11-20, LP12T12-40 & $223.4-236.3$ & 24.72 & -1.84 & 15.96 \\
\hline & qPW-10 & 10 & LP4T5-8, LP4T5-134 & $433.2-447.2$ & 12.04 & 1.154 & 6.04 \\
\hline & qPW-11-1 & 111 & LP1T4-87, LP1T4-90 & $0.01-17.9$ & 12.57 & -0.944 & 4.76 \\
\hline & & & LP1T2-43, LP1T2-45, LP2T6-75 & & & & \\
\hline & qPW-11-2 & 211 & LP11T12-9, LP11T12-15 & $44.65-74.83$ & 13.43 & -1.167 & 5.04 \\
\hline & qPW-15 & 15 & LP7T7-38, LP7T7-37 & $119.9-134.8$ & 12.84 & -1.220 & 6.49 \\
\hline & qPW-33 & 33 & LP3T6-5, LP5T8-22 & $224.9-236.5$ & 22.96 & 1.654 & 13.51 \\
\hline \multirow[t]{4}{*}{ Cocoon shell rate } & qRC-6 & 6 & LP6T1-42, LP6T1-11 & $1105.7-1121.3$ & 14.10 & 2.01 & 11.13 \\
\hline & $\mathrm{qRC}-12$ & 12 & LP11T7-38, LP11T7-23 & $2.01-9.5$ & 17.70 & -2.43 & 16.56 \\
\hline & $\mathrm{qRC}-3$ & 21 & LP12T10-24, LP12T12-25, LP12T12-22 & $89.8-106.3$ & 15.17 & -2.05 & 12.14 \\
\hline & qRC-4 & 23 & LP2T3-42, LP1T4-119, LP2T2-55 & $87.8-101.2$ & 22.51 & -2.42 & 19.69 \\
\hline
\end{tabular}



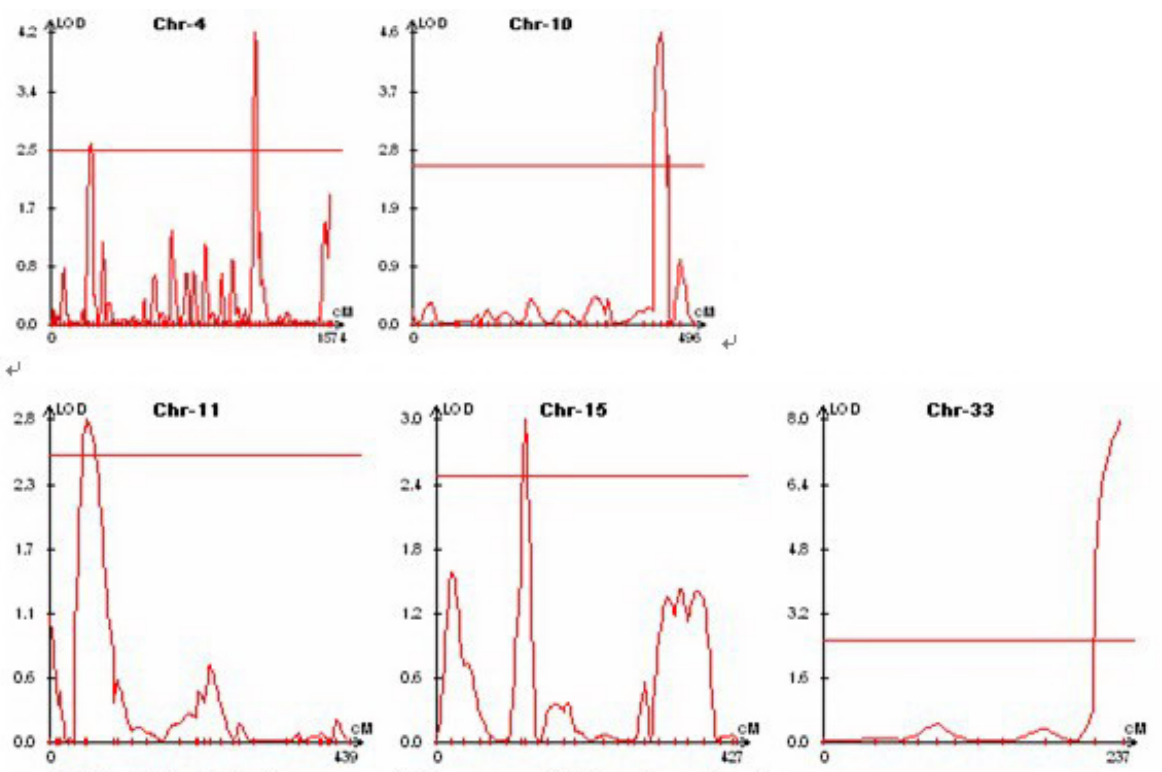

Figure 1. Quantitative trait loci of whole cocoon weight by composite interval mapping.
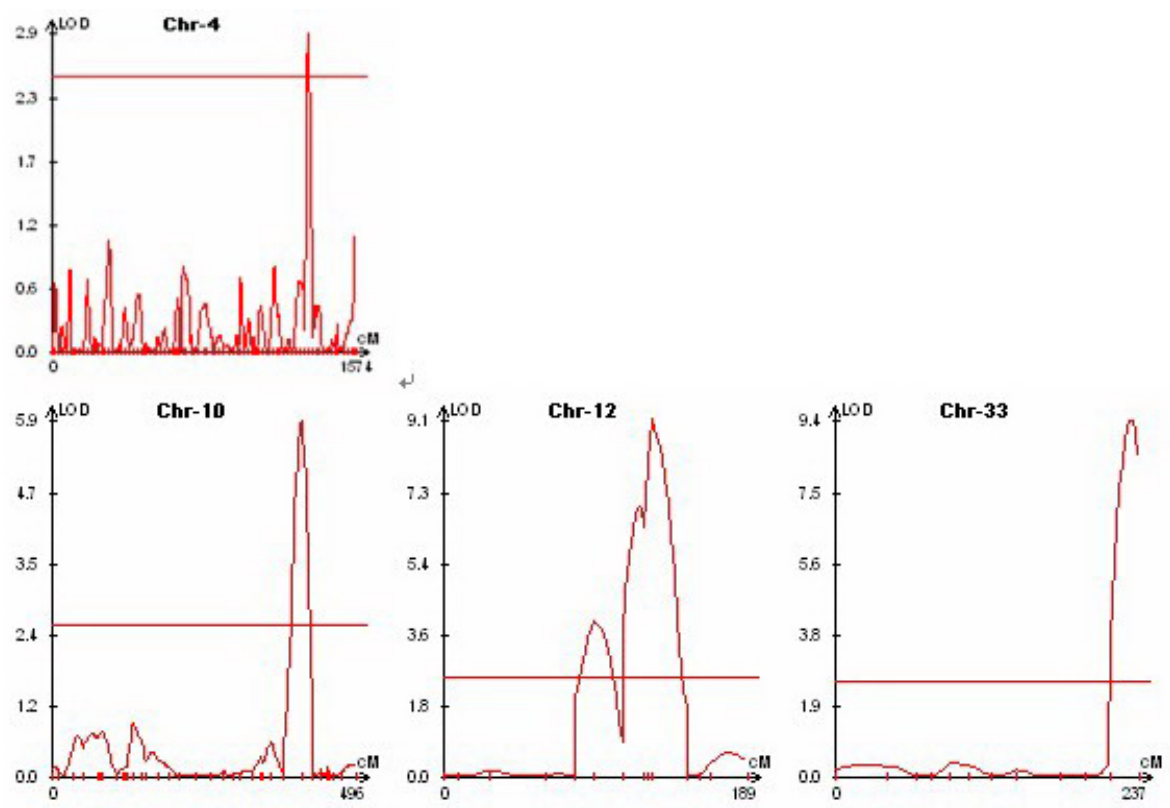

Figure 2. Quantitative trait loci of whole cocoon shell weight by composite interval mapping. 

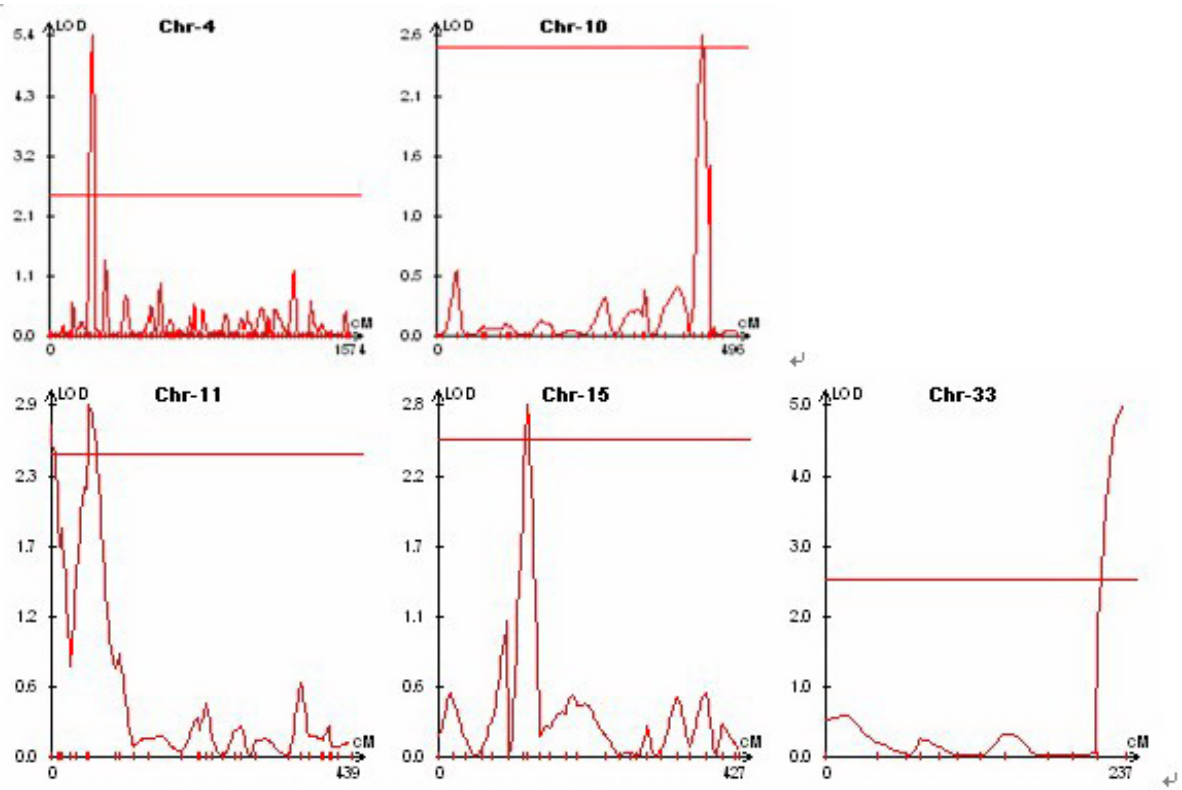

Figure 3. Quantitative trait loci of pupa weight by composite interval mapping.
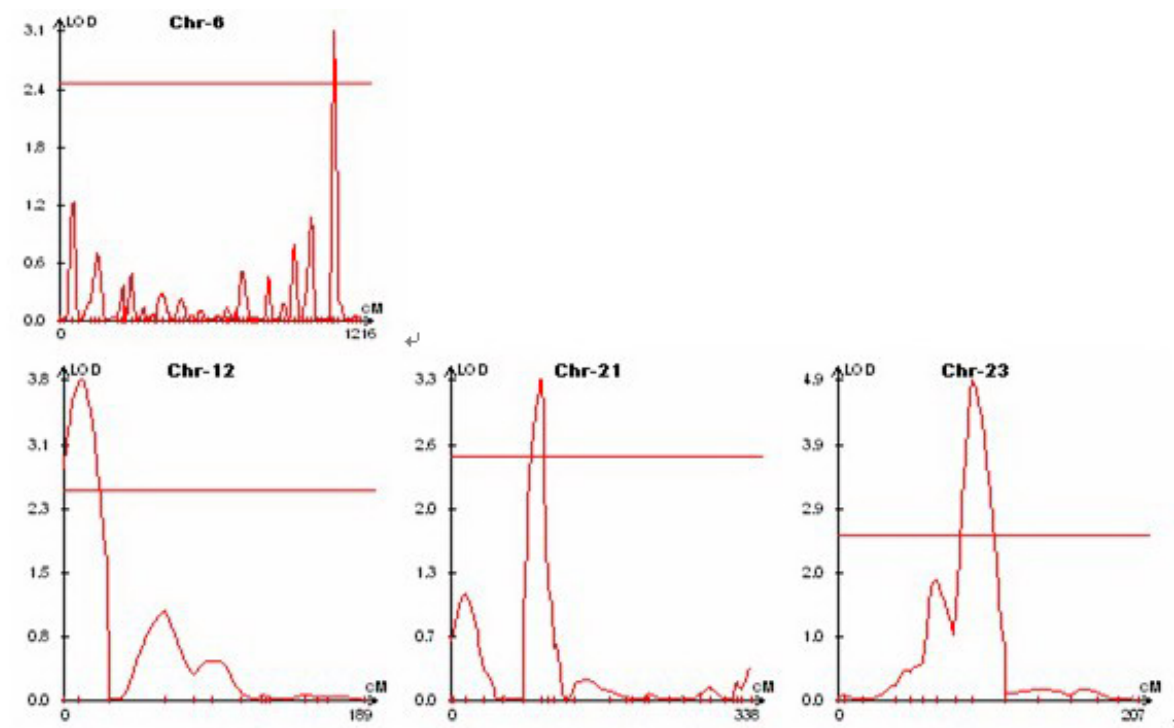

Figure 4. Quantitative trait loci of cocoon shell rate by composite interval mapping. 


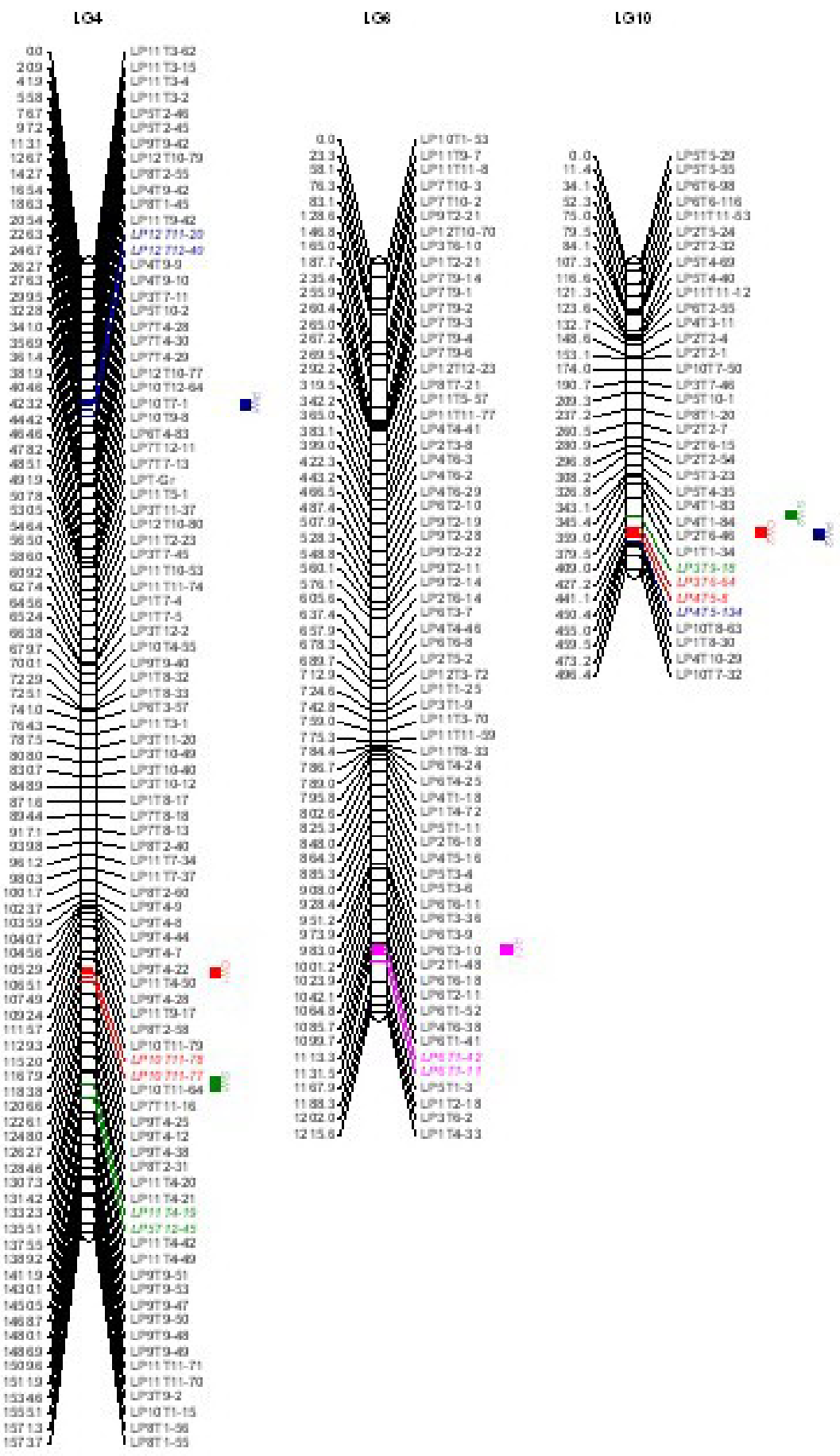

Figure 5. Continued on next page. 

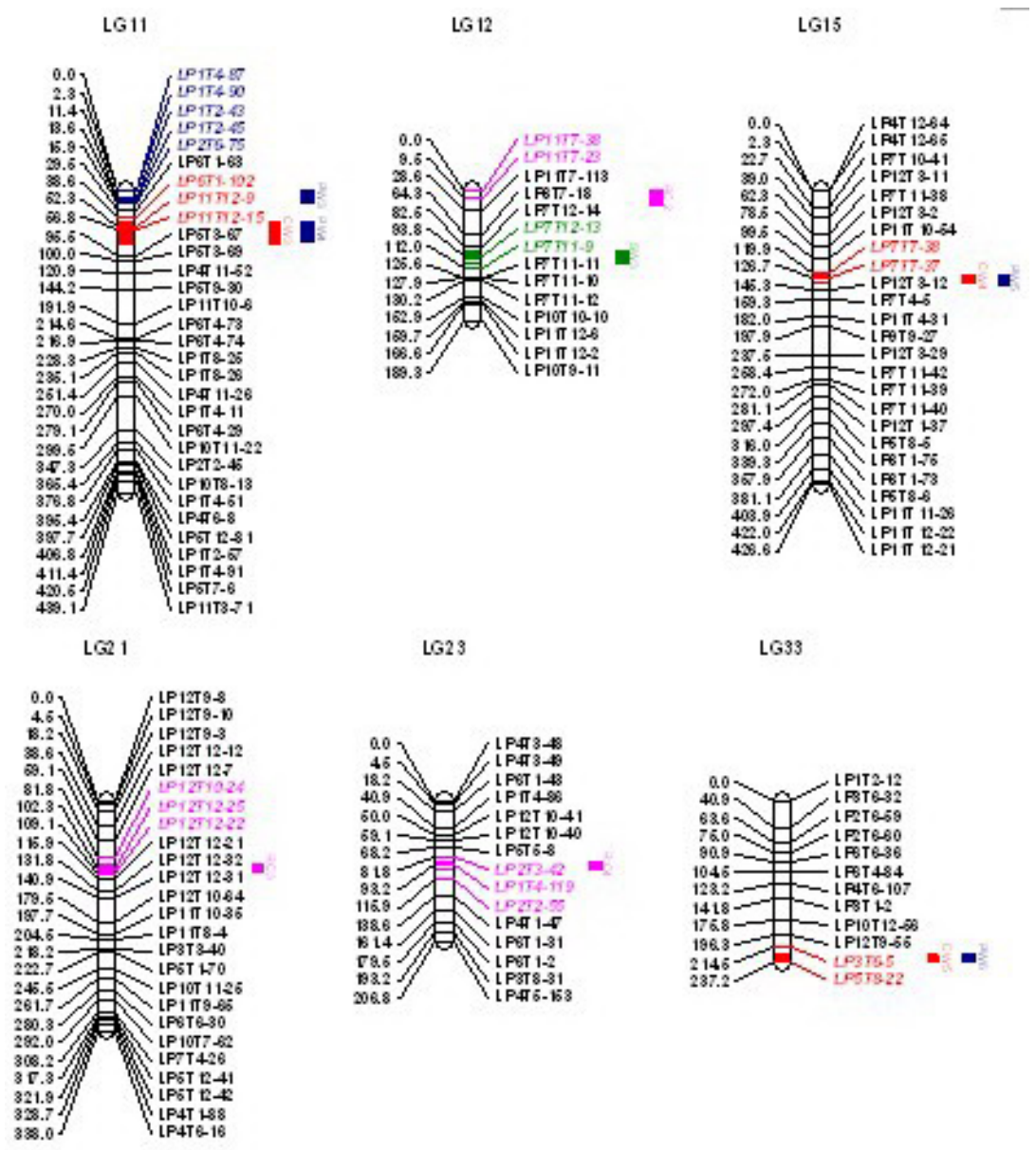

Figure 5. Location of putative quantitative trait loci for whole cocoon weight, cocoon shell right, pupa weight, and cocoon shell rate traits in AFLP linkage map of Bombyx mori. CW represents whole cocoon weight; SW represents cocoon shell weight; PW represents pupa weight; RC represents cocoon shell rate.

\section{Whole cocoon weight}

Five QTLs of whole cocoon weight were detected (Figure 1), which accounted for $51.0 \%$ of the variation. The ratio of contribution of individual QTLs ranged from 4.7 to $23.2 \%$, and their additive effect was from -1.803 to $2.560 \mathrm{~g}$. The ratios of contributions of qCW-10 and qCW-33 on 10 and 33 linkage groups were 10.9 and $23.2 \%$, respectively, which were major QTLs with additive effects of 1.830 and $2.563 \mathrm{~g}$, respectively. The ratio of contribution of the other three QTLs ranged from 4.7 to $6.2 \%$, and their additive effect was from -1.299 to $1.571 \mathrm{~g}$. 


\section{Cocoon shell weight trait}

Four QTLs of cocoon shell weight were detected (Figure 2), which explained 73.69\% of the variation, and their additive effect ranged from -3.581 to $7.27 \mathrm{~g}$. The ratios of contribution of qSW-12, qSW-10 and qSW-33 on 12, 10 and 33 linkage group were 10.68, 17.76 and $37.08 \%$, respectively, which were main QTLs, whose additive effect was 4.19, 5.67 and $7.27 \mathrm{~g}$, respectively. The ratio of contribution of qSW-4 was $8.172 \%$ with additive effects of $-3.581 \mathrm{~g}$.

\section{Pupa weight trait}

Six QTLs of pupa weight were detected (Figure 3) in total, accounting for $51.80 \%$ of the variation. Their additive effects ranged from -1.840 to $1.654 \mathrm{~g}$. Among them, the contribution rates of qPW-33 and qPW-4 to the traits on 33rd and 4th linkage groups are 13.51 and $15.69 \%$, respectively. As major QTLs, their additive effect was 1.654 and $-1.840 \mathrm{~g}$, respectively.

\section{Cocoon shell rate trait}

Four QTLs of cocoon shell rate were detected (Figure 4), accounting for $59.52 \%$ of the variation. The contribution rate of individual QTL was between 11.13 and $19.69 \%$, whose additive effect was within -2.43-2.01\%. Among them, the contribution ratios of qRC-6, qRC-21, qRC12 and $\mathrm{qRC}-23$ to the traits on the 6th, 21st, 12th, and 23rd linkage groups were 11.13, 12.14, 16.56 , and $19.69 \%$, respectively, as they are major QTLs, and their additive effects are negative.

\section{DISCUSSION}

According to classical silkworm genetic theory, four major economic cocoon traits in the silkworm, that is, whole cocoon weight, cocoon shell weight, pupa weight, and cocoon shell rate, are controlled by a polygene. In this experiment, on the logarithm of odds value more than 2.5 condition, five QTLs of whole cocoon weight, four QTLs of cocoon shell weight, six QTLs of pupa weight, and four QTLs of cocoon shell rate trait scatter nine linkage groups depending on the segregative population of $\mathrm{BC}_{1}$. From the results, there are eleven major effect QTLs whose contribution rate is over 10\%. Among them, two QTLs account for over $20 \%$ of the contribution rate, controlling whole cocoon weight (CW-33), cocoon shell weight (SW-33), and these two QTLs could be very helpful in improving cocoon trait. In the present experiment, all QTLs detected have a significant confidence interval, so these QTLs could be very helpful in silkworm molecular-assisted selection. Consequently, the different additive effect of QTLs could be utilized closely linked marker to combine and obtain high-yield species. The additive effects of nine QTLs are positive and those of ten QTLs are negative. These results imply that silkworm breeding is very difficult to achieve a breeding target. This QTL map of silkworm cocoon trait establishes a good foundation for further molecular marker-assisted breeding.

Although the separation traits of the backcross generation $\left(\mathrm{BC}_{1}\right)$ are less than that of the second hybrid generation $\left(\mathrm{F}_{2}\right)$, the analysis of the separation traits of backcross generation is easier than that of $\mathrm{F}_{2}$. This is because the inheritance of silkworm females behaves in a completely linkage manner. In the present study, a backcrossed population $\left(\mathrm{BC}_{1}\right)$ derived from a cross between the inbred strain $\mathrm{C}_{100}$ and Dazao was employed to construct the mapping population. This linkage map 
constructed from the entire silkworm genome, but the individual of mapping population was insufficient to find overall QTLs. The variances explained for QTLs of whole cocoon weight, cocoon shell weight, pupa weight, and cocoon shell rate were 51.0, 73.69, 51.80, and 59.52\%, respectively. The result implies that some QTLs of silkworm for cocoon traits were undetected in the present linkage map. For example, sex chromosome of silkworm was very important for cocoon trait by classical silkworm genetics, because the major genes of cocoon shell weight and whole cocoon weight were on the sex chromosome or their inheritance characteristic showed sex-linked inheritance. The sex chromosome could not be identified in this linkage map, so the QTLs of cocoon traits in this study could not be compared with previous QTLs determined in classical silkworm genetics. On the one hand, for the detection of more silkworm QTLs, improving the density of linkage map, for example, increase mapping population and join multiple molecular marker would facilitate detection of other QTLs. On the other hand, morphological markers located on every chromosome of the traditional silkworm linkage map could be joined with molecular markers to detect the others, so that the silkworm marker-assisted selection method will be improved.

\section{ACKNOWLEDGMENTS}

Research supported by the National 973 Program of China (\#2005CB121000), Program of the Education Department of Sichun Provence (\#2005A105) and Program of China West Normal University (\#05B040). The authors are grateful to Dr. Zhang Zheng-sheng for his assistance with the software.

\section{REFERENCES}

Bradshaw JE, Hackett CA, Pande B, Waugh R, et al. (2008). QTL mapping of yield, agronomic and quality traits in tetraploid potato (Solanum tuberosum subsp. tuberosum). Theor. Appl. Genet. 116: 193-211.

Goldsmith MR (1991). The Bombyx mori genome-mapping project. Sericologia 31: 145-155.

Hansen C, Yi N, Zhang YM, Xu S, et al. (2005). Identification of QTL for production traits in chickens. Anim. Biotechnol. 16: 67-79.

Li B, Lu C, Zhou ZY and Xiang ZH (2000). Construction of silkworm RAPD molecular linkage map. Yi. Chuan Xue. Bao. (Acta Genet. Sin.) 27: 127-132.

Miao XX, Xub SJ, Li MH, Li MW, et al. (2005). Simple sequence repeat-based consensus linkage map of Bombyx mori. Proc. Natl. Acad. Sci. U. S. A. 102: 16303-16308.

Rothschild MF, Hu ZL and Jiang Z (2007). Advances in QTL mapping in pigs. Int. J. Biol. Sci. 3: 192-197.

Saiki RK, Scharf S, Faloona F, Mullis KB, et al. (1992). Enzymatic amplification of beta-globin genomic sequences and restriction site analysis for diagnosis of sickle cell anemia. Biotechnology 24: 476-480.

Sax K (1923). The association of size differences with seed-coat pattern and pigmentation in Phaseolus vulgaris. Genetics 8: 552-560.

Tan YD, Wan C, Zhu Y, Lu C, et al. (2001). An amplified fragment length polymorphism map of the silkworm. Genetics 157: 1277-1284.

Tanksley SD, Young ND, Paterson AH and Bonierbale MW (1989). RFLP mapping in plant breeding: new tools for an old science. Bio/Technology 7: 257-264.

Thoday JM and Boam TB (1961). Regular responses to selection I. Description of responses. Genet. Res. 2: 161-176.

Vos P, Hogers R, Bleeker M, Reijans M, et al. (1995). AFLP: a new technique for DNA fingerprinting. Nucleic Acids Res. 23: 4407-4414.

Wan CL and Tan YD (1999). An improved method of AFLP. J. Nanjing Normal University (Natural Science Edition) 22: 88-91. Wang SC, Basten CJ and Zeng ZB (2002). QTL Cartographer. Version 2.0. Department of Statistics, North Carolina State University, Raleigh.

Williams JG, Kubelik AR, Livak KJ, Rafalski JA, et al. (1990). DNA polymorphisms amplified by arbitrary primers are 
useful as genetic markers. Nucleic Acids Res. 18: 6531-6535.

Xia QY, Zhou ZY, Lu C and Xiang ZH (1998). Molecular phylogenetic study on the racial differentiation of Bombyx mori by random amplified polymorphic DNA (RAPD) markers (in Chinese). Acta Entomol. Sin. 41: 32-40.

Zabeau M and Vos P (1992). Selective Restriction Fragment Amplification: a General Method for DNA Fingerprinting. Publication 0534858 A1. Bulletin 93/13. European Patent Office, Munich.

Zeng ZB, Kao CH and Basten CJ (1999). Estimating the genetic architecture of quantitative traits. Genet. Res. 74: 279-289.

Zhang L, Qian M, Dai FY, Zhao AC, et al. (2008). A dense linkage map of the silkworm (Bombyx mori) based on AFLP markers. Acta Entomol. Sin. 51: 246-257.

Zhou Y (1958). General Entomology. 2nd edn. High Education Publication House, Beijing. 